\title{
Fiat lux
}

\section{Technical advances in biophotonics allow scientists to get a better picture of the cell}

J ust as engineers learnt to harness the power of electrons in transistors and microchips-which revolutionized modern society-physicists and biologists are now learning how to tame photons. Their work is generating exciting new possibilities for optical microscopy, with a resolution and level of detail hitherto undreamt of. Biophotonics is a new and exciting research field that is already delivering substantial dividends in a wide range of applications from fundamental medical research to diagnosis, therapy and surgery.

For a long time the resolution of optical microscopes had been considered to be insufficient for most biological research applications. Consequently, optical microscopes were largely confined to classrooms and pathology laboratories until innovations, such as fluorescence microscopy and laser confocal microscopy, began to bring biophotonics back into the light. In combination with computerassisted image analysis and improved sample preparation methods (Hunter, 2005), various other technological advances have provided even higher levels of resolution and accuracy. These have allowed optical techniques to capitalize on the advantage that they always had over X-ray crystallography or electron microscopy-the ability to observe biochemical processes in real time.

$\mathrm{P}$ robably the most important step in the resurgence of biophotonics was the breaking of the Abbe limit, named after the German theoretical physicist Ernst Abbe (1840-1905). The Abbe limit states that no microscope can resolve optical details smaller than half the wavelength of the light used for illumination. Fortunately, the Abbe limit only applies to conventional microscopy that observes far-field images from a distance; by exploiting interference patterns and other effects, it is possible to achieve a much finer spatial resolution beyond the wavelength of light (see sidebar). Generally these effects are not directly visible, as in classical optical microscopy, but require computer processing to generate an image of the object.

\section{Probably the most important step in the resurgence of biophotonics was the breaking of the Abbe limit...}

Another historical problem that the latest optical techniques are able to overcome is the study of samples in vivo without the need for staining or other methods of sample preparation to provide the necessary contrast. "The whole problem with biological cells is that they are really not light-absorbing objects, and so can't be seen easily under a microscope [without preparation]," said Michael Feld, Director of the George R. Harrison Spectroscopy Laboratory at the Massachusetts Institute of Technology (Cambridge, MA, USA).

One way of reconstructing images with greater resolution than would be possible with standard optical microscopy is to use the information from the light refracted by the sample, rather than the light absorbed by it. This task is made easier by the fact that although cells do not absorb light very well, they do refract it; each molecule on and within a cell bends light in a characteristic way that can be detected by comparison to a reference beam. The basic idea behind the technique is interferometry, in which two electromagnetic waves-in this case light beams-interfere to create a new wave. Given that the reference beam of light has a known direction, amplitude and wavelength, a computer can calculate how the subject has refracted the analytical beam from the interference patterns, which provides much higher resolution than conventional light images. This, in turn, reveals what the object is made of and how it looks.

In his research, Feld uses a particular application of interferometry, so-called quantitative phase contrast imaging. "It converts the phase shift to an absorption change, which can then be studied with microscopic techniques," he explained. It also provides more detailed information about the image. As Feld has noted, the incredible depth of resolution afforded by interferometry, combined with the ability to study living cells, allows him to perform real-time measurements of processes down to a scale of just $1 \AA$ - which is comparable with electron microscopy. By using this method, Feld studies the dynamics of the membranes of red blood cells in great detail (Popescu et al, 2005). "I like to think of it as the hydrogen atom of cells," Feld explained his choice of the red blood cell. "It just has a membrane, some internal scaffold, and a homogeneous distribution of haemoglobin."

\section{...by exploiting interference patterns and other effects, it is possible to achieve a much finer spatial resolution beyond the wavelength of light}

But it is not only the simplicity of red blood cells that make them interesting - they are also implicated in several diseases, most notably malaria and sickle-cell anaemia. The latter appears to have evolved as a side effect of an imperfect solution to provide partial protection against malaria, particularly in children, although the molecular mechanisms involved are still not fully understood. Feld and his colleagues have been studying how changes in a red blood cell alter the mechanical behaviour of its membrane 


\section{SHINING LIGHT ON CELLS}

Optical microscopy uses the fact that light shone through or onto an object is diffracted or bent; the image created by the light beams is therefore not continuous, but broken up into peaks and troughs of intensity created by interference between rays of light. A classical optical microscope focuses a magnifying lens system on these interferences to create a distinct image. However, the ability of such a microscope to resolve fine details is limited both by the properties of light and the refractive material of the lenses. The maximum resolution thus achievable is approximately half the wavelength of the light, or around $200 \mathrm{~nm}$-closer than that and details become too fuzzy to observe. This led to Abbe's diffraction limit of microscopy, which seemed to shut the door on optical microscopy as a tool for delving deeper into molecular details. Fortunately, it turned out that Abbe's limit only applies to microscopes that create a directly observable image. This is sometimes called far-field microscopy because the light from the object travels through a magnifying lens system over distances many magnitudes greater than the size of the object or the area under examination.

The Abbe law does not make any stipulation about the ability to resolve detail at distances closer than half the wavelength of light. In that case, the direct link between the object and the observer's eye is cut—such techniques analyse or recreate the image indirectly, usually through computer analysis. This might involve near-field techniques in which light is measured close to the object. Alternatively, light can be projected from longer distances, providing it creates interference patterns at close range that can then be observed and analysed.

In addition, some modern microscopic techniques also exploit the fact that molecules exhibit fluorescence effects, whereby they emit light at distinctive wavelengths after stimulation with light of a different wavelength. Again, this does not provide direct images, but it does allow position and size to be accurately determined at resolutions as small as $1.2 \mathrm{~nm}$, which is almost 200 times above the Abbe limit, and strays into the territory of electron microscopy. Furthermore, fluorescence avoids the need for staining the samples in order to illuminate them, given that organic molecules are usually poor at absorbing light.

Another technique to avoid the staining problem is quantitative phase contrast imaging, which is the highest resolution imaging technique developed. Similar to its use in transmission electron microscopy, it analyses the diffraction patterns that molecules create when light is shone through them, instead of observing the light that is absorbed and retransmitted. This technique generates much more information about the object than many other methods, because it is not confined to the measurement of physical dimensions. It also provides detailed information about the concentrations and numbers of individual elements, as revealed by the characteristic phase and amplitude of diffracted light waves. Scientists use it to track cellular processes in real time, but it also has huge potential for more accurate and high-throughput medical diagnostic methods.

during malarial infection and sickle-cell disease, because such changes can influence immune responses. "As a red blood cell ages, its mechanical properties change, and eventually it is discarded when it can't function properly any longer," Feld explained.

He also emphasized that it was impossible to study the mechanical properties of cell membranes in this way with previously available techniques of any kind because they lacked the combination of real-time imaging and spatial and temporal resolution. Feld also uses the same technique to track neuronal signals, which manifest as changes of about $1 \mathrm{~nm}$ in the diameter of a neuron. "We hope in turn this will be extended to studying the electrical function of whole simple organisms," he said.

$\mathrm{T}$ he high-resolution technique Feld is using is still largely confined to basic research; however, another technique, optical coherence tomography (OCT), which analyses light reflected back from the object-albeit with lower resolution-has already found its way into the clinic. "Think of this as an analogue to ultrasound, but based on infrared light," explained Stephen Boppart, Director of the Mills Breast Cancer Institute (Urbana, IL, USA). "[W]e collect reflections, and using the coherence properties of light, we can gaze out and determine precisely where those reflections come from and use that information to reconstruct the image." Although the technique can only penetrate tissue up to $3 \mathrm{~mm}$, it is sufficient to analyse small tissue samples in real time, and is already used in cancer diagnosis and surgery (Fig 1).

In the absence of real-time analysis methods, surgeons usually remove a substantial amount of healthy tissue to reduce the chance of leaving malignant cells behind; it is not always practical to do biopsies on marginal tissue because results might take 40 minutes or more to obtain.
To overcome this time lag, the Mills Breast Cancer Institute uses a portable OCT scanner to identify tumour cells during surgery. "At the moment we're able to view the surgical margin with our OCT system and do that very rapidly to determine if that margin is clear of tumour cells," said Boppart. This rapid diagnostic system allows surgeons to leave more healthy tissue, which, in the case of breast cancer surgery, is obviously desirable, especially for cosmetic reasons. It is even more important in brain surgery to minimize the loss of healthy neurons and cognitive functions.

\section{...optical coherence tomography (OCT), which analyses light reflected back from the object-albeit with lower resolution-has already found its way into the clinic}

The idea behind OCT is that all cell types, including the various categories of tumour cells, produce distinctive coherence patterns that can be readily detected. In addition, the scanning process can be automated to make life easier for the surgeon and to reduce errors. "We recognise that when trying to scan large areas of tissue very quickly, a large amount of data is produced that a human operator (such as a surgeon) cannot spend the time to look through," said Boppart. "So that's why we've developed automatic identification algorithms that can try and characterise the different tissue types."

The same technique also has the potential to help cancer diagnosis through image-guided keyhole biopsies. Although they still need to be tested clinically, needles incorporating a tiny camera that generate OCT images as they penetrate tissue have been tested successfully in the laboratory, according to Feld. Inside the needle is a glass fibre approximately $125 \mu \mathrm{m}$ in diameter-just slightly thicker than an average human hair-which both radiates light and collects reflections. If successful, it will largely avoid the need for biopsies in diagnosis and could further reduce the number of false negatives. Small cancer nodules less than $1 \mathrm{~cm}$ in size are quite frequently missed during biopsies, but could be found by using an OCT-guided needle.

There is also the possibility-now being evaluated at the Mills Breast Cancer 
Institute-of extending image-guided needles to the screening of healthy patients with a family history or genetic susceptibility to breast cancer. This would involve ductoscopy, where such a needle would be inserted into breast ducts to screen for inflammation and hyperplasia-swelling through abnormal cell proliferationwhich often precedes the formation of malignant tumours.

Raman spectroscopy is another technique that is being developed for screening tumours. Conventional spectroscopy techniques, such as vibrational spectroscopy, exploit the fact that molecules absorb light at different wavelengths according to their atomic structure. Raman spectroscopy, by contrast, detects differences in the way light is scattered, which is more convenient to measure in living tissue. "Essentially we obtain a spectroscopic signature made up of a mixture of DNA, proteins and lipids," said Simon Herrington, Professor of Pathology at the University of St Andrews in Scotland, UK. Similarly to OCT, this technique allows scientists to distinguish cell types and also to identify some of the early stages of cancer development even before hyperplasia has developed.

Herrington and colleagues (Jess et al, 2007) have shown that Raman spectroscopy can identify infection by human papilloma virus (HPV) by revealing characteristic spectral peaks originating from viral DNA. Again, this has clinical implications; for example, infection with HPV, in particular a variant called HPV16, is known to increase the risk of cervical cancer.

The power of OCT and spectroscopy in diagnostics lies neither in high-resolution images, nor in analysing biochemical processes as they occur, but in the ability to accurately distinguish between different tissue and cell types by measuring their optical properties. Conversely, the quantitative phase contrast imaging used by Feld does achieve very high resolutions and real-time measurements, although Feld has not used it to monitor live processes so far.

$\mathrm{S}$ till, one of the greatest advantages of optical techniques is the possibility to study cellular processes in vivo, as well as biochemical reactions in vitro. This requires not only high spatial resolution, but also temporal resolution, because interactions between molecules can occur over very short distances and timescales. Indeed, it was optical techniques that lifted

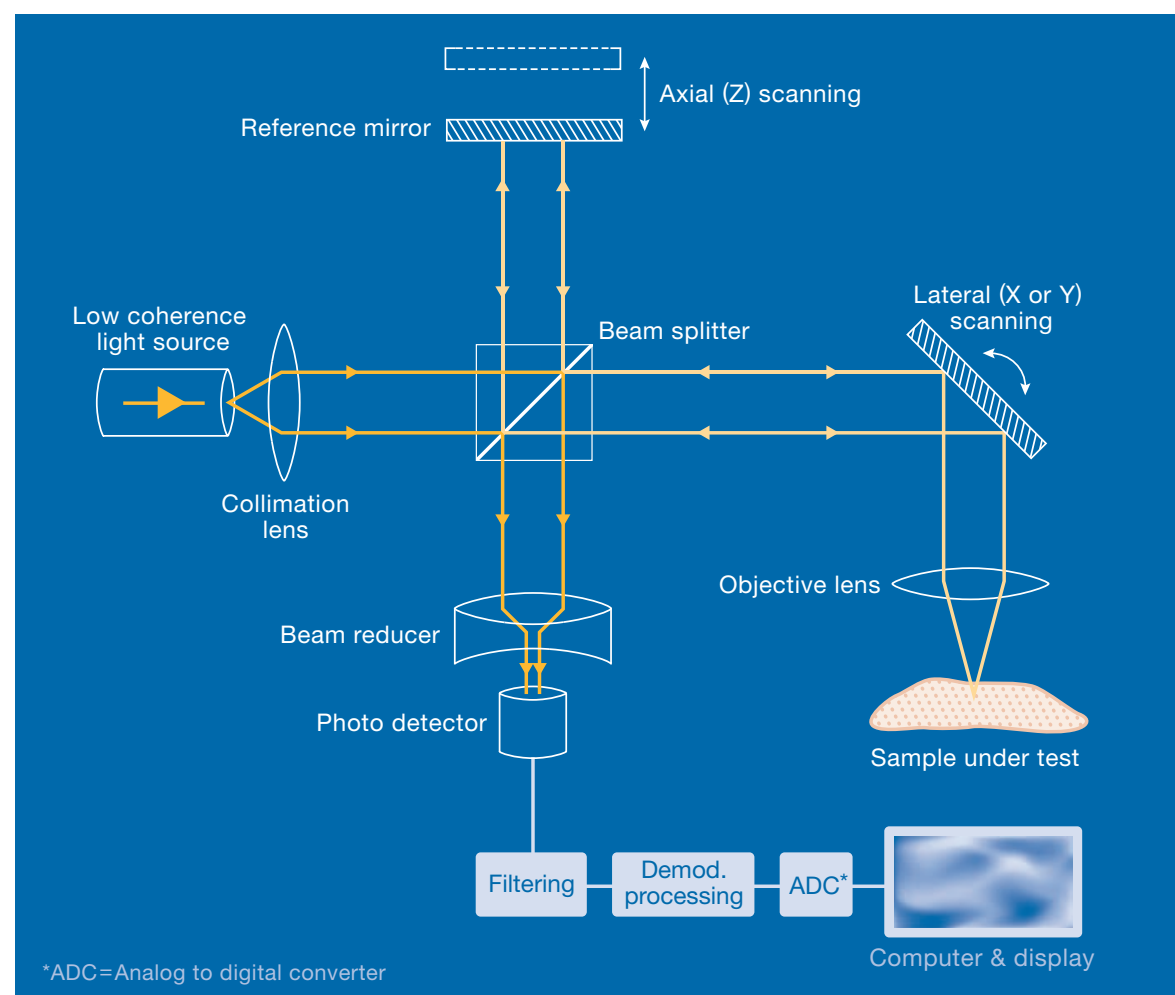

Fig 1 | Typical optical setup of single point optical coherence tomography. Scanning the light beam on the sample enables non-invasive cross-sectional imaging up to $3 \mathrm{~mm}$ in depth with micrometre resolution. Credit: Wikipedia.

the veil on various intimate molecular processes, according to Ben Fabry, head of the biophysics group at Erlangen University in Germany, who studies how protein receptors behave as they hook up to cells. "There is no other way of measuring biochemical reactions in the living cell," he said.

\section{The power of OCT and spectroscopy in diagnostics lies [...] in the ability to accurately distinguish between different tissue and cell types by measuring their optical properties}

Fabry's work has mostly been in vitro, triggering artificial vibrations in cells to investigate their mechanical properties. The technique his team has developed uses magnetic beads approximately $5 \mu \mathrm{m}$ in diameter, which are coated with ligands through which they are attached to a cell surface. By applying suitable magnetic fields, the scientists can trigger oscillations of varying strengths and frequencies, which, in turn, cause vibrations in the cell that can be measured optically. Fabry's team, together with colleagues in the USA, have used this technique to study the dynamics of the cytoskeleton in living cells (Bursac et al, 2007; Raupach et al, 2007).

Fabry is also using the technique to study the mechanical properties of cells involved in cancer metastasis, which is still poorly understood. The ability of malignant cells to migrate through tissues and set up new colonies depends on their mechanical flexibility, which allows them to squeeze through tight intercellular spaces. A growing understanding of the role of mechanical features in cancer cells could help to identify the crucial cells involved-not all tumour cells have this mechanical flexibility. "It's conceivable [that] we could get a biopsy from a patient and characterise particularly aggressive cancer cells," Fabry said. "There are so-called hypothelial cancer stem cells that can migrate more efficiently." In effect, such cells start to behave collectively almost like a liquid, flowing through healthy tissue in 
search of suitable locations to metastasize and start new colonies.

The new generation of optics is also being applied to the study of autoimmune conditions. One of the main challenges to understanding how the immune system can go wrong has been to work out how regulatory $T$ cells $\left(T_{R E C}\right)$ normally suppress immune attacks on the body's own tissue, and how this can fail. By using two-photon laser scanning microscopy, it is now possible to investigate these processes because the technique allows the observation of crucial events in vivo (see sidebar). But the use of fluorescence has two further advantages: the source provides its own light, which is convenient, and it is highly specific, enabling scientists to 'light up' and observe individual molecules.

Qizhi Tang, Jeffrey Bluestone and Max Krummel at the University of California San Francisco, USA, have used the technique to study the behaviour and movement of $\mathrm{T}_{\text {REG }} \mathrm{S}$ in diabetic mice (Tang et al, 2006). This work has revealed some surprises about $\mathrm{T}_{\mathrm{REC}} \mathrm{S}$ - for example, that such cells move around much faster than had been expected, even when not triggered by an antigen. However, the most interesting finding was that $\mathrm{T}_{\mathrm{REG}} \mathrm{S}$ do not tackle pathogenic $T$ cells directly, but instead inhibit their ability to interact with so-called dendritic cells, which activate pathogenic $T$ cells by presenting them with an antigen derived from the target for attack. Normally, such a target might be a pathogen or a cell compromised by a virus, but in autoimmune diseases the antigen comes from healthy cells. $T_{\text {REG }} \mathrm{s}$ inhibit such undesirable priming of pathogenic $\mathrm{T}$ cells by stopping them from interacting with dendritic cells and coming into contact with the antigen. Although this investigation is still at the 'pure research' stage, insights about the role and behaviour of $\mathrm{T}_{\mathrm{REG}} \mathrm{S}$ could be a starting point for therapies to treat autoimmune diseases.

As the field of biophotonics matures, it will generate more innovations for both research and clinical applications; there are already many exciting uses for the new generation of microscopes in diagnosis and therapy beyond cancer. But probably even more exciting is the fact that these new techniques allow scientists to shine light on the manifold mysteries of the cell at a level of detail only previously possible with electron microscopy - an illuminating prospect.

\section{REFERENCES}

Bursac P, Fabry B, Trepat X, Lenormand G, Butler JP, Wang N, Fredberg JJ, An SS (2007) Cytoskeleton dynamics: fluctuations within the network. Biochem Biophys Res Commun 355: 324-330

Hunter $\mathrm{P}$ (2005) The bigger picture. EMBO Rep 6: 1113-1116

Jess PRT, Smith DDW, Mazilu M, Dholakia K, Riches AC, Herrington CS (2007) Early detection of cervical neoplasia by Raman spectroscopy. Intern J Cancer (in press)

Popescu G, Ikeda T, Best CA, Badizadegan K, Dasari RR, Feld MS (2005) Erythrocyte structure and dynamics quantified by Hilbert phase microscopy. J Biomed Opt 10: 060503

Raupach C, Zitterbart DP, Mierke CT, Metzner C, Muller FA, Fabry B (2007) Stress fluctuations and motion of cytoskeletal-bound markers. Phys Rev E Stat Nonlin Soft Matter Phys 76: 011918

Tang Q, Adams JY, Tooley AJ, Bi M, Fife BT, Serra P, Santamaria P, Locksley RM, Krummel MF, Bluestone JA (2006) Visualizing regulatory T cell control of autoimmune responses in nonobese diabetic mice. Nat Immunol 7: 83-92

Philip Hunter

doi:10.1038/sj.embor.7401078 